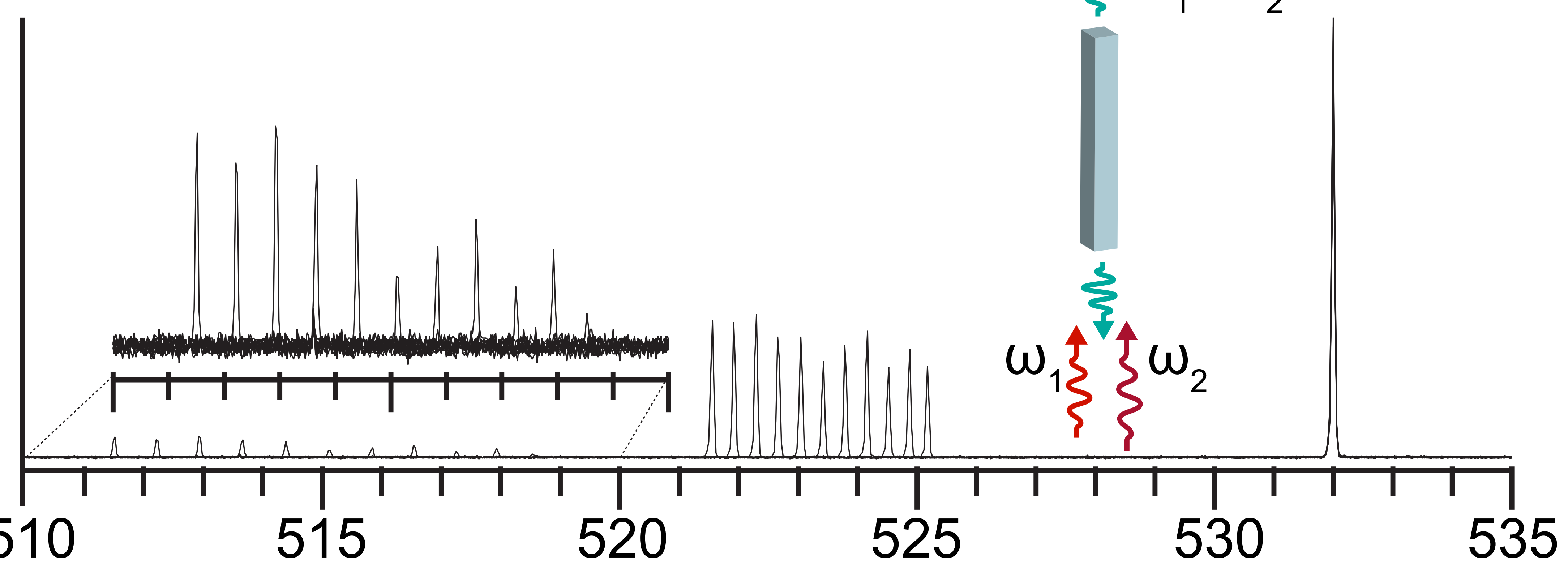




\title{
Hot Brownian thermometry and cavity-enhanced harmonic generation with nonlinear optical nanowires
}

\author{
Bennett E. Smith ${ }^{\mathrm{a}, 1}$, Paden B. Roder ${ }^{\mathrm{b}, 1}$, Xuezhe Zhou ${ }^{\mathrm{b}}$, Peter J. \\ Pauzauskie ${ }^{\mathrm{b}, \mathrm{c}, *}$ \\ ${ }^{a}$ Department of Chemistry, University of Washington, Seattle, Washington, 98195 \\ ${ }^{b}$ Material Science \& Engineering Department, Seattle, University of Washington, \\ Washington, 98195 \\ ${ }^{c}$ Fundamental \& Computational Sciences Directorate, Pacific Northwest National \\ Laboratory, Richland, WA 94720
}

\begin{abstract}
The non-linear response of nanoscale materials is affected by phase-matching conditions that are critically dependent on temperature. It has remained a persistent challenge to measure the temperature of individual nonlinear optical nanostructures in situ. Here, we measure the temperature of individual $\mathrm{KNbO}_{3}$ nanowires in an optical trap through analysis of the Brownian dynamics. Additionally, we show Fabry-Pérot-type cavity enhancement of second harmonic generation in one-dimensional potassium niobate nanowires (KNNW) using a tunable, continuous wave (CW), near-infrared (NIR) trapping laser. A second co-aligned CW NIR laser is used to extend the range of visible emission through sum frequency generation.

Keywords: optical trap, potassium niobate, nonlinear, second harmonic, sum frequency, Brownian motion
\end{abstract}

\section{Introduction}

The nonlinear response of materials to intense electric fields were only realized after the advent of the laser. The first reported example was second harmonic generation (SHG) of a ruby laser in crystalline quartz [1]. Since then

\footnotetext{
${ }^{*}$ Corresponding author: peterpz@uw.edu

${ }^{1}$ These authors contributed equally to this work.
}

Preprint submitted to Chemical Physics Letters

September 15, 2015 
5 researchers in the field of nonlinear optics have demonstrated harmonics as high as $n=221$ [2]. Harmonic generation has a significant utility in converting lasers beams of frequency $\omega$ into beams with frequency of $n \omega$ where $n$ is an integer. Other commonly used nonlinear effects include parametric processes such as sum frequency generation (SFG), difference frequency generation (DFG), and opti-

10 cal parametric oscillation (OPO), or non-parametric processes like two-photon absorption (TPA) and stimulated Raman scattering [3. Applications of nonlinear processes vary significantly and include laser frequency modulation [1, 4], two-photon luminescence [5], vibrational spectroscopy [6], two-photon medical imaging [7, nonlinear photodynamic therapy [8, 9], and information technology 15 10.

Nanostructures have gained in popularity as nonlinear optical materials [11, 12, 13, 14, 15], notably for biomedical theranostics [16]. Applications include drug delivery [17, photothermal [18] and photodynamic [19, 20] therapy, and imaging [21]. Nonlinear nanoparticles are especially advantageous for biomed20 ical imaging [22] as they allow for the use of near infrared (NIR) light which penetrates deeper into living tissue than visible light due to lower absorption 23. One-dimensional structures have the advantage of a large surface area providing a scaffold for adsorption and conjugation. Potassium niobate is a popular nonlinear material [24] and has even had nonlinear effects demonstrated from 25 optically trapped nanowires 25]. However, knowing the temperature of $\mathrm{KNbO}_{3}$ nanomaterials under NIR illumination is important not only for phase-matching conditions but also if it is to be used in vivo for theranostic applications given the low temperature threshold $\left(\sim 42^{\circ} \mathrm{C}[26]\right)$ for cellular damage. Here, the Brownian dynamics of optically trapped KNNWs are analyzed in order to extract temperatures. Nonlinear processes, including SHG and SFG, are observed from the trapped KNNWs and Fabry-Pérot type cavity enhanced SHG is demonstrated using a tunable NIR laser which suggests the possibility of synthetically tuning the lengths and diameters of nanowire cavities for increased harmonic conversion [12, 13, 14. 


\section{Experimental}

Monoclinic $\mathrm{KNbO}_{3}$ nanowires were grown hydrothermally using a previouslyreported method [27. Briefly, $12.6 \mathrm{~g}$ of $\mathrm{KOH}$ was dissolved in $15 \mathrm{~mL} \mathrm{DI} \mathrm{H}_{2} \mathrm{O}$ and sonicated for 5 minutes. After adding $0.87 \mathrm{~g}$ of niobium metal powder to this solution it was placed into a teflon-lined autoclave and heated to $150^{\circ} \mathrm{C}$ for $40 \quad 15$ hours.

Samples for scanning electron microscopy (SEM), x-ray diffraction (XRD), and Raman spectroscopy were prepared by evaporating an aqueous suspension onto a clean silicon wafer. Scanning electron micrographs were acquired on a JEOL JSM-7000F scanning electron microscope in secondary electron imaging mode with a $10.0 \mathrm{kV}$ accelerating voltage and $10.0 \mathrm{~mm}$ working distance. Bright field and select area electron diffraction (SAED) images were taken on a FEI Tecnai G2 F20 at an accelarating voltage of $200 \mathrm{keV}$. SAED images were taken with a camera length of $490 \mathrm{~mm}$. XRD measurements were performed on a Bruker D8 Discover with GADDS XRD system using $\mathrm{Cu} \mathrm{K}_{\alpha}$ radiation. Raman spectra were obtained using a home-built setup using a Coherent Compass 532 $\mathrm{nm}$ SLM laser at $10 \mathrm{~mW}$ power. The laser was focused onto the sample using a Mitotoyo 50x long working distance objective $(\mathrm{NA}=0.55)$. Backscattered light was dispersed onto a Princeton liquid nitrogen-cooled silicon CCD using an Acton 500i SpectraPro spectrograph.

Near-edge X-ray absorption fine structure (NEXAFS) spectroscopy was perfomed on the Advance Light Source beamline 5.3.2.2 at Lawrence Berkeley National Laboratory. Samples were prepared by evaporating a $2 \mu \mathrm{L}$ suspension of nanowires onto a lacey carbon TEM grid and placing the TEM in the beamline under 0.33 atmopheres of helium. Soft x-rays $(250-800 \mathrm{eV})$ were focused onto the sample with a spot size of $31 \mathrm{~nm}$ using $25 \mathrm{~nm}$ Fresnel zone plates. The oxygen K-edge was observed in the energy window of $525-550 \mathrm{eV}$.

Optical trapping of individual KNNWs was accomplished with a home-built system (Fig. 3). A $330 \mathrm{~mW}$ Thorlabs $975 \mathrm{~nm}$ diode laser, $4 \mathrm{~W}$ SpectaPhysics 1064 nm DPSS laser, and 10 W Sahajanad Laser Technology 1020-1040 nm 
tunable DPSS Yb:YAG disk laser were co-aligned, expanded, and focused into a chamber using a Nikon 100x oil immersion objective (NA=1.25). SHG and SFG signals were collected using the same objective and detected with the same spectrometer used for the Raman measurements. Trapping chambers consisted of $75 \times 25 \times 1 \mathrm{~mm}$ glass slide and \#1 coverslip with a droplet of NW suspension 70 and imaging spacer (Grace Bio-Labs) confined between them.

\section{Results \& Discussion}

The crystal structure of the as-synthesized KNNWs was determined using characterization techniques including: SAED, XRD, Raman spectroscopy, and scanning transmission x-ray microscopy. The hydrothermal method used has

75 been characterized extensively [27] and the current results support a monoclinic phase. SEM images clearly show the orthogonal cross sections of KNNWs signifying highly crystalline growth (Fig. 1 1 ) with an average edge length measuring $152 \pm 49 \mathrm{~nm}$, which lies below the single-mode waveguide cutoff for $975 \mathrm{~nm}$ in $\mathrm{KNbO}_{3}: 229 \mathrm{~nm}$. However, the inhomogeneous contrast found in the bright field TEM image of an individual KNNW (Fig. 10) indicates a significant amount of extended crystallographic defects (Fig. 11;) including stacking faults and twinning planes. The corresponding SAED pattern (Fig. 1 $1 \mathrm{~d}$ ) confirms a monoclinic phase with a [100] growth direction. Faint lines can also be seen in the SAED pattern which agrees with stacking faults observed in brightfield micrographs.

85 X-ray diffraction (Fig. 1 p) and Raman spectra (Fig. 1f) also support a monoclinic phase. Weak signals can be seen in the XRD plot at $38^{\circ}$ and $70^{\circ}$ which are not attributable to $\mathrm{KNbO}_{3}$ and are likely from residual $\mathrm{Nb}$ metal precursor. However, analysis of these peaks using the Scherrer equation 28] gives a particle size $>15 \mathrm{~nm}$ which are not observed in TEM brightfield images or SAED

90 patterns from individual KNNWs, meaning there is no $\mathrm{Nb}$ metal within the KNNWs themselves. Since 1-dimensional $\mathrm{KNbO}_{3}$ structures are easily identified optically during laser trapping experiments, $\mathrm{Nb}$ precursor material can be excluded as a contributing factor for heating of trapped KNNWs. 
The chemical bonding environment surrounding the niobate ion can be probed with scanning transmission x-ray microscopy (STXM). A suspended KNNW was imaged using scanning transmission x-ray microscopy (Fig. 2a) and a linescan of the nanowire was used to extract x-ray absorption in the energy range of $520-580 \mathrm{eV}$. Observation of the $\mathrm{O}$ K-edge at $530 \mathrm{eV}$ shows a slight deviation from the simple $\mathrm{O} 1 \mathrm{~s} \rightarrow \mathrm{O} n \mathrm{p}$ transition. The XAS absorption profile of $\mathrm{KNbO}_{3}$ has been explained previously [29] through the hybridization of $\mathrm{O} 2 \mathrm{p}$ and with several different $\mathrm{Nb}$ orbitals including $4 \mathrm{~d}\left(\mathrm{t}_{2 g}\right), 4 \mathrm{~d}\left(\mathrm{e}_{g}\right), 5 \mathrm{~s}$, and $5 \mathrm{p}$. The spectrum measured from our material (Fig. 2p) matches well with the previous report well.

The electric fields generated within an optically-trapped KNNW is of sufficient intensity that the material will respond nonlinearly, described by the second-order polarization:

$$
\mathbf{P}(t)=\epsilon_{0} \chi^{(2)} \mathbf{E}^{2}(t)
$$

Here $\epsilon_{0}$ is the permittivity of free space, $\chi^{(2)}$ is the second-order nonlinear susceptibility, and $\mathbf{E}(t)$ is the electric field. Gases, liquids, amorphous solids, and many crystal structures display inversion symmetry and, therefore, have a vanishing $\chi^{(2)} . \mathrm{KNbO}_{3}$, however, is a non-centrosymmetric crystal which will demonstrate second-order nonlinear effects. If a field consisting of two frequency components, expressed as $\mathbf{E}(t)=\mathbf{E}_{1} \cos \left(\omega_{1} t-k x\right)+\mathbf{E}_{2} \cos \left(\omega_{2} t-k x\right)$, 110 is incident on a nonlinear material, it can be seen that, following Eq. 1, the resultant fields will include second harmonic frequencies $\left(2 \omega_{1}, 2 \omega_{2}\right)$, the sum frequency $\left(\omega_{1}+\omega_{2}\right)$, and the difference frequency $\left(\omega_{1}-\omega_{2}\right)$. In the current experiment, co-aligned lasers of different frequencies are used to demonstrate simultaneous SHG and SFG in trapped KNNWs. In one experiment the 975 and $1064 \mathrm{~nm}$ lasers were used to trap the same NW producing their respective second harmonic signals at 487.5 and $532 \mathrm{~nm}$ in addition to the sum frequency signal at $509 \mathrm{~nm}$ (Fig. 4 4 ). Figure $4 \mathrm{~b}$ shows the potential of tunable SFG from an optically trapped KNNW. Eleven spectra are overlaid with each spectrum showing SHG from the $1064 \mathrm{~nm}$ laser, SHG from the NIR tunable laser set to 
120 a particular wavelength, and the SFG signal from the tuned and 1064 lasers. It can be inferred from these observations that there is also radiation generated at wavelengths corresponding to the difference frequencies: $11.7 \mu \mathrm{m}(106 \mathrm{meV})$, the difference between $1064 \mathrm{~nm}+975 \mathrm{~nm}$ and $24.7-46.1 \mu \mathrm{m}(27-50 \mathrm{meV})$, the difference between the tunable NIR laser $+1064 \mathrm{~nm}$. However, the estimated 125 power for SHG and SFG signals, accounting for grating and detector efficiencies, is $10^{-14} \mathrm{~W}$ and, assuming a similar efficiency for DFG, the mid-infrared powers would be over an order of magnitude less than visible powers. Even with water's high absorption in the mid-infrared, solvent heating due to DFG is unlikely due to neglible powers generated. Figure 4 . shows the relative intensities of SHG polarized parallel and perpendicular to the $1064 \mathrm{~nm}$ fundamental beam. The ratio of polarization for $\mathrm{SHG}(\sim 10)$ indicates that the visible luminescence from a trapped KNNW is able to maintain a significant amount of coherence which could be beneficial for applications where lasing is required on a micro- and nano-scale.

Optical trapping of a single nanostructure with parallel end facets also permits the observation of Fabry-Pérot resonances, depending on the particle geometry and wavelength of light. In this case, the second harmonic of the tunable laser lies in the range 510 - $520 \mathrm{~nm}$ while the cavity length is approximately 4 micrometers. The mode spacing is expressed [30]:

$$
\Delta \lambda=\frac{\lambda^{2}}{4 L\left[\lambda \frac{\partial n}{\partial \lambda}-n\right]}
$$

135 the dispersion of the refractive index, and $L$ is the length of the NW. Using reported 31] values for the optical properties of $\mathrm{KNbO}_{3}$, the predicted mode spacing is $6.5 \mathrm{~nm}$ which matches well with the experimental value of $5.8 \mathrm{~nm}$. The width of the SHG signal can also be used to comment on the quality factor 140 of the KNNW based on the formula $Q=\omega_{0} / \delta \omega$, giving a value of $\sim 80$.

Temperatures of particles within an optical trap are often ignored based on assumptions of neglible absorption. But the significant temperature dependence of phase-matching for nonlinear materials requires a better understanding of the 
heat generated in these materials under intense illumination. It has been demonstrated that back focal plane interferometry of forward-scattered laser light can be used to monitor the Brownian motion of a trapped NW. [32] In these experiments the forward-scattered laser light is collected and focused onto a quadrant photodiode where the voltage-to-displacement calibration factor is found using a piezo-oscillation method [33. Once the calibration factor is determined the experimental diffusion coefficient can be found by fitting the calibrated QPD power spectrum 34. The temperature of the trapped NW can then be determined by equating the experimental diffusion coefficient to the hot Brownian motion diffusion coefficient of a heated particle in a locally non-isothermal environment [35, 36] and solving for the particle's temperature. Temperatures of trapped KNNWs were found using this method and are compared to temperatures of $\mathrm{SiO}_{2}$ beads at identical trapping powers in Fig. 6. The bandgap of $\mathrm{KNbO}_{3}$ lies in the ultraviolet region of the spectrum [37. so one would expect little one- or two-photon absorption from the NIR wavelengths used to trap the NW. However, crystal defects can enhance optical absorption within the lattice resulting in heat generation. 38. The inhomogeneous contrast seen in the KNNW bright-field TEM micrograph (Fig. 1p and c) indicates lattice distortion which is further supported by the SAED pattern where the faint linear streaks between spots characteristic of large numbers of extended crystallographic defects. Experimental temperatures can be used in conjunction with recent theory 32 for heating of finite cylinders in order to estimate the imaginary refractive, $k$ of a trapped KNNW. By assuming a circular cylinder with a length of $4.12 \mu \mathrm{m}$ and diameter equal to the edge length of the KNNW square cross section (150 $\mathrm{nm}$ ), it is found that $k \approx 0.0005$, which corresponds to an equivalent absorption coefficient of $\alpha \approx 60 \mathrm{~cm}^{-1}$.

\section{Conclusion}

We have demonstrated that the use of a tunable, 1020-1040 nm, continuouswave NIR trapping source with hydrothermally synthesized potassium niobate 
nanowires provides tunable second harmonic generation which can then by used to extract an effective refractive index through Fabry-Pérot mode spacings. 175 Additionally, the range of tunability of the nonlinear emission in the visible region can be extended by introducing a second, co-aligned NIR laser source resulting in sum frequency generation. Given the potential for application of these nonlinear materials in theranostic nanomedicine, the heat generated by nanoscale particles in the intense fields necessary for nonlinear effects could have a significant impact. Temperature measurement through analysis of particle Brownian motion shows an increase from room temperature to $\sim 60^{\circ} \mathrm{C}$ at an irradiance of approximately $10 \mathrm{MW} / \mathrm{cm}^{2}$ which we attribute to crystallographic defects evidenced in the contrast from bright field TEM and SAED patterns. Future efforts could exploit the narrow linewidth and tunability of the visible SHG from individual KNNWs, for example, to selectively pump narrowband absorbers like rare-earths or photosensitizers for photodynamic therapy.

\section{Acknowledgement}

This research was made possible by a grant from the Air Force Office of Scientific Research Young Investigator Program (contract \#FA95501210400) and start-up funding from the University of Washington. B.E.S. acknowledges support from a NIH T32 training grant (T32CA138312). P.B.R. thanks the NSF for a Graduate Research Fellowship under grant number DGE-1256082. XRD and TEM were conducted at the University of Washington NanoTech User Facility, a member of the NSF National Nanotechnology Infrastructure Network ${ }_{195}$ (NNIN). XAS was performed at the Advanced Light Source at Lawrence Berkeley National Laboratory. The authors also thank E. James Davis for donating an optical spectrometer with $\mathrm{LN}_{2}$-cooled detector, A. L. David Kilcoyne for help XAS measurements, and K. Kroy for constructive feedback on the hot Brownian motion analysis. 
205 215

\section{References}

[1] P. A. Franken, A. E. Hill, C. W. Peters, G. Weinreich, Generation of optical 口 harmonics, Physical Review Letters 7 (4) (1961) 118-119. doi:10.1103/ PhysRevLett.7.118

[2] Z. Chang, A. Rundquist, H. Wang, M. M. Murnane, H. C. Kapteyn, Gen-

[3] R. W. Boyd, Chapter 1 - the nonlinear optical susceptibility, in: R. W. Boyd (Ed.), Nonlinear Optics (Third Edition), Academic Press, Burlington,

[4] J. A. Giordmaine, R. C. Miller, Tunable coherent parametric oscillation in $\mathrm{LiNbO}_{3}$ at optical frequencies, Physical Review Letters 14 (24) (1965) 973-976. doi:10.1103/PhysRevLett.14.973

[5] W. Kaiser, C. G. B. Garrett, Two-photon excitation in $\mathrm{CaF}_{2}: \mathrm{Eu}^{2+}$, Physeration of coherent soft $\mathrm{X}$ rays at $2.7 \mathrm{~nm}$ using high harmonics, Physical Review Letters 79 (16) (1997) 2967-2970. doi:10.1103/PhysRevLett.79. 2967. 2008, pp. 1-67.

ical Review Letters 7 (6) (1961) 229-231. doi:10.1103/PhysRevLett.7. 229.

[6] Z. Lu, A. Karakoti, L. Velarde, W. Wang, P. Yang, S. Thevuthasan, H.-f. Wang, Dissociative binding of carboxylic acid ligand on nanoceria surface in aqueous solution: A joint in situ spectroscopic characterization and firstprinciples study, The Journal of Physical Chemistry C 117 (46) (2013) 24329-24338. doi:10.1021/jp4068747.

[7] G. Thomas, J. van Voskuilen, H. C. Gerritsen, H. J. C. M. Sterenborg, Advances and challenges in label-free nonlinear optical imaging using twophoton excitation fluorescence and second harmonic generation for cancer research, Journal of Photochemistry and Photobiology B: Biology 141 (2014) 128-138. doi:10.1016/j.jphotobiol.2014.08.025. 
[8] A. V. Kachynski, A. Pliss, A. N. Kuzmin, T. Y. Ohulchanskyy, A. Baev, J. Qu, P. N. Prasad, Photodynamic therapy by in situ nonlinear photon conversion, Nature Photonics 8 (6) (2014) 455-461. doi:10.1038/ nphoton.2014.90.

[9] M.-C. Hsieh, C.-H. Chien, C.-C. Chang, T.-C. Chang, Aggregation induced photodynamic therapy enhancement based on linear and nonlinear excited FRET of fluorescent organic nanoparticles, Journal of Materials Chemistry B 1 (18) (2013) 2350-2357. doi:10.1039/C3TB00345K.

235 [10] E. Garmire, Nonlinear optics in daily life, Optics Express 21 (25) (2013) 30532. doi:10.1364/0E.21.030532.

[11] S. Roke, G. Gonella, Nonlinear Light Scattering and Spectroscopy of Particles and Droplets in Liquids, Annual Review of Physical Chemistry 63 (1) (2012) 353-378. doi:10.1146/annurev-physchem-032511-143748.

[12] J. C. Johnson, H. Yan, R. D. Schaller, P. B. Petersen, P. Yang, R. J. Saykally, Near-Field Imaging of Nonlinear Optical Mixing in Single Zinc

a Oxide Nanowires, Nano Letters 2 (4) (2002) 279-283. doi:10.1021/ nl015686n.

[13] J. C. Johnson, H. Yan, P. Yang, R. J. Saykally, Optical Cavity Effects in ZnO Nanowire Lasers and Waveguides, The Journal of Physical Chemistry B 107 (34) (2003) 8816-8828. doi:10.1021/jp034482n.

[14] J. C. Johnson, K. P. Knutsen, H. Yan, M. Law, Y. Zhang, P. Yang, R. J. Saykally, Ultrafast Carrier Dynamics in Single ZnO Nanowire and Nanoribbon Lasers, Nano Letters 4 (2) (2004) 197-204. doi:10.1021/nl034780w

[15] P. Peng, D. J. Milliron, S. M. Hughes, J. C. Johnson, A. P. Alivisatos, R. J. Saykally, Femtosecond Spectroscopy of Carrier Relaxation Dynamics in Type II CdSe/CdTe Tetrapod Heteronanostructures, Nano Letters 5 (9) (2005) 1809-1813. doi:10.1021/n10511667. 
[16] B. E. Smith, P. B. Roder, X. Zhou, P. J. Pauzauskie, Nanoscale materials

[17] A. S. Angelatos, B. Radt, F. Caruso, Light-responsive polyelectrolyte/gold nanoparticle microcapsules, The Journal of Physical Chemistry B 109 (7) (2005) 3071-3076. doi:10.1021/jp045070x.

[18] C. Guo, S. Yin, H. Yu, S. Liu, Q. Dong, T. Goto, Z. Zhang,

[20] D. K. Chatterjee, L. S. Fong, Y. Zhang, Nanoparticles in photodynamic therapy: An emerging paradigm, Advanced Drug Delivery Reviews 60 (15)

[21] R. Ladj, T. Magouroux, M. Eissa, M. Dubled, Y. Mugnier, R. L. Dantec, C. Galez, J.-P. Valour, H. Fessi, A. Elaissari, Aminodextran-coated potassium niobate $\left(\mathrm{KNbO}_{3}\right)$ nanocrystals for second harmonic bio-imaging, Colloids and Surfaces A: Physicochemical and Engineering Aspects 439 (2013)

[22] P. Pantazis, J. Maloney, D. Wu, S. E. Fraser, Second harmonic generating (SHG) nanoprobes for in vivo imaging, Proceedings of the National

1. Academy of Sciences 107 (33) (2010) 14535-14540. doi:10.1073/pnas. 1004748107.

[23] R. Weissleder, A clearer vision for in vivo imaging, Nature Biotechnology 19 (4) (2001) 316-317. doi:10.1038/86684. 
[24] D. Fluck, P. Gnter, Efficient generation of CW blue light by sum-frequency mixing of laser diodes in $\mathrm{KNbO}_{3}$, Optics Communications 136 (34) (1997) 257-260. doi:10.1016/S0030-4018(96)00693-1.

[25] Y. Nakayama, P. J. Pauzauskie, A. Radenovic, R. M. Onorato, R. J. Saykally, J. Liphardt, P. Yang, Tunable nanowire nonlinear optical probe, Nature 447 (7148) (2007) 1098-1101. doi:10.1038/nature05921.

[26] J. A. Dickson, B. E. Oswald, The sensitivity of a malignant cell line to hyperthermia $\left(42^{\circ} \mathrm{C}\right)$ at low intracellular $\mathrm{pH}$., British Journal of Cancer 34 (3) (1976) 262-271.

[27] S. Kim, J.-H. Lee, J. Lee, S.-W. Kim, M. H. Kim, S. Park, H. Chung, Y.-I. Kim, W. Kim, Synthesis of monoclinic potassium niobate nanowires that are stable at room temperature, Journal of the American Chemical Society 135 (1) (2013) 6-9. doi:10.1021/ja308209m.

[28] B. D. Cullity, Elements Of X-Ray Diffraction, Addison-Wesley Publishing Company, Inc., 1956.

[29] L. Douillard, F. Jollet, C. Bellin, M. Gautier, J. P. Duraud, The electronic structure of KNbO3: an XPS and XAS study, Journal of Physics: Condensed Matter 6 (27) (1994) 5039. doi:10.1088/0953-8984/6/27/013.

[30] J. van der Ziel, Effect of fabry-perot interference on second harmonic generation in a GaAs plate, IEEE Journal of Quantum Electronics 12 (7) (1976) 407-411. doi:10.1109/JQE.1976.1069179.

[31] N. Umemura, K. Yoshida, K. Kato, Phase-Matching Properties of KNbO_3 口 in the Mid-Infrared, Applied Optics 38 (6) (1999) 991. doi:10.1364/AO. 38.000991 .

[32] P. B. Roder, B. E. Smith, E. J. Davis, P. J. Pauzauskie, Photothermal heating of nanowires, The Journal of Physical Chemistry C 118 (3) (2014) 1407-1416. doi:10.1021/jp4078745 
[33] S. F. Tolić-Nørrelykke, E. Schäffer, J. Howard, F. S. Pavone, F. Jülicher,

[37] I. Grinberg, D. V. West, M. Torres, G. Gou, D. M. Stein, L. Wu, G. Chen, E. M. Gallo, A. R. Akbashev, P. K. Davies, J. E. Spanier, A. M. Rappe,

[38] T. J. Magee, J. Peng, J. Bean, Microscopic Defects and Infrared Absorption in Cadmium Telluride, physica status solidi (a) 27 (2) (1975) 557-564. doi:10.1002/pssa.2210270226. 

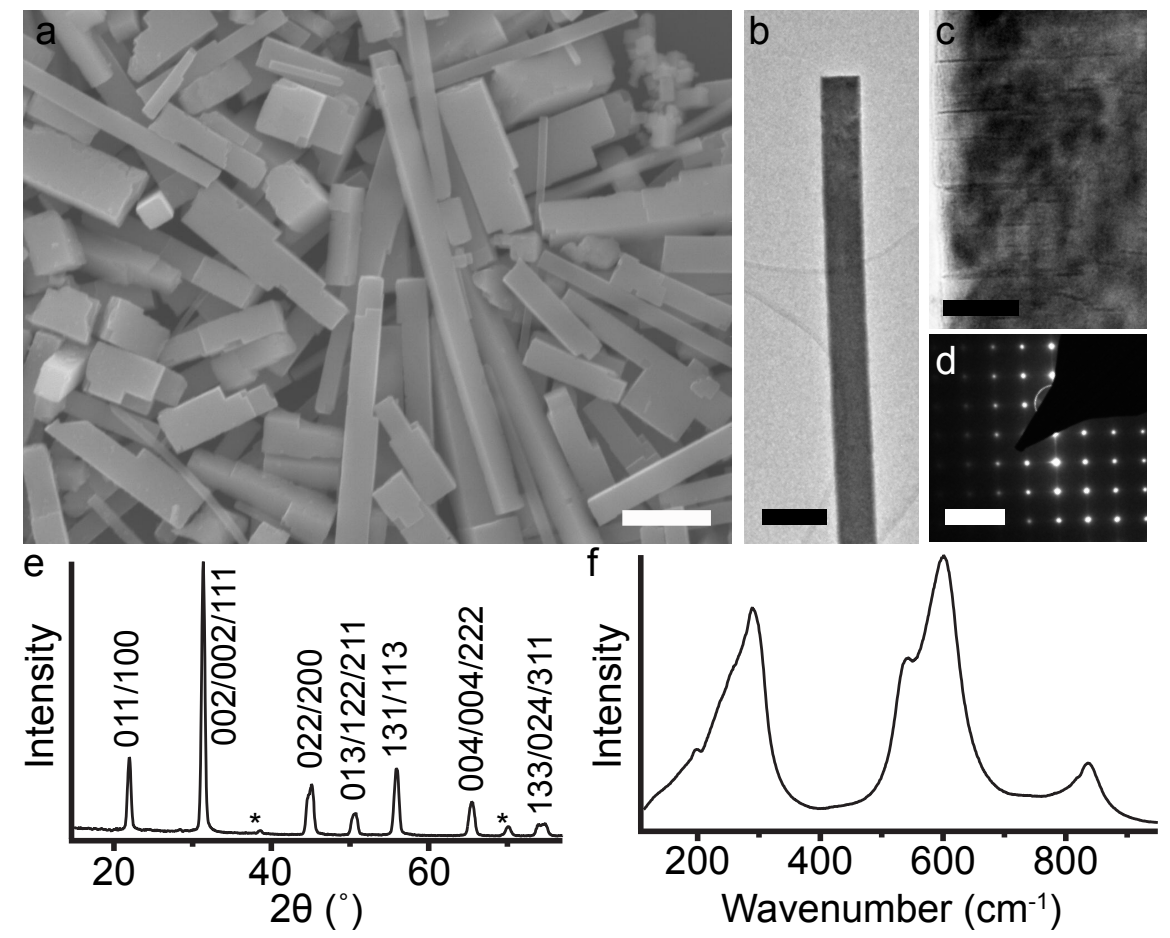

Figure 1: Characterization of hydrothermally grown nanowires. a) Scanning electron micrograph of KNNWs demonstrating square cross-sections. Scalebar $=500 \mathrm{~nm}$. b) Transmission electron micrograph of a single KNNW. Scalebar $=300 \mathrm{~nm}$. c) Higher magnification of KNNW in $\mathrm{b}$ to show extended planar defects in a KNNW. Scalebar $=30 \mathrm{~nm}$. d) Select area electron diffraction pattern of the KNNW in b. Scalebar $=5 \mathrm{~nm}^{-1}$ e) X-ray diffraction of a $\mathrm{KNbO}_{3}$ powder sample with crystal planes indicated. Asterisk denotes peaks not associated with $\mathrm{KNbO}_{3}$ and are likely from $\mathrm{Nb}$ metal precursor. f) Raman scattering from a $\mathrm{KNbO}_{3}$ powder sample. 

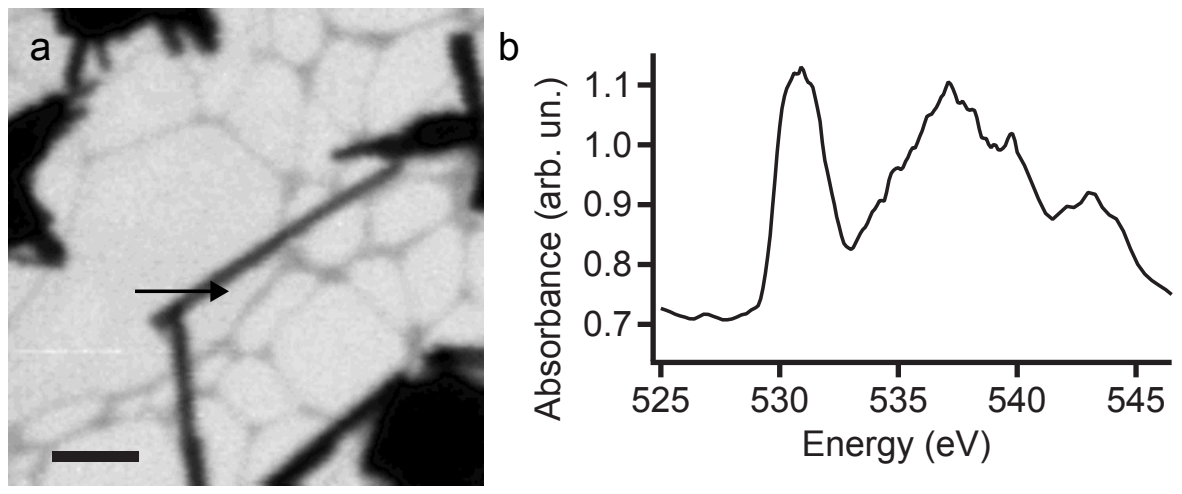

Figure 2: (a) Scanning transmission x-ray micrograph of KNNWs suspended on a lacey carbon TEM grid. The arrow indicates the line drawn with the linescan used to determine the spectrum in panel (b). X-ray energy for the micrograph is $540 \mathrm{eV}$. Scalebar $=1 \mu \mathrm{m}$. (b) Near edge X-ray absorption fine structure (NEXAFS) of the O K-edge from the monoclinic KNNW linescan in (a) demonstrating the effect of hybridization of the $\mathrm{O} 2 \mathrm{p}$ orbitals with the $\mathrm{Nb}$ orbitals. 


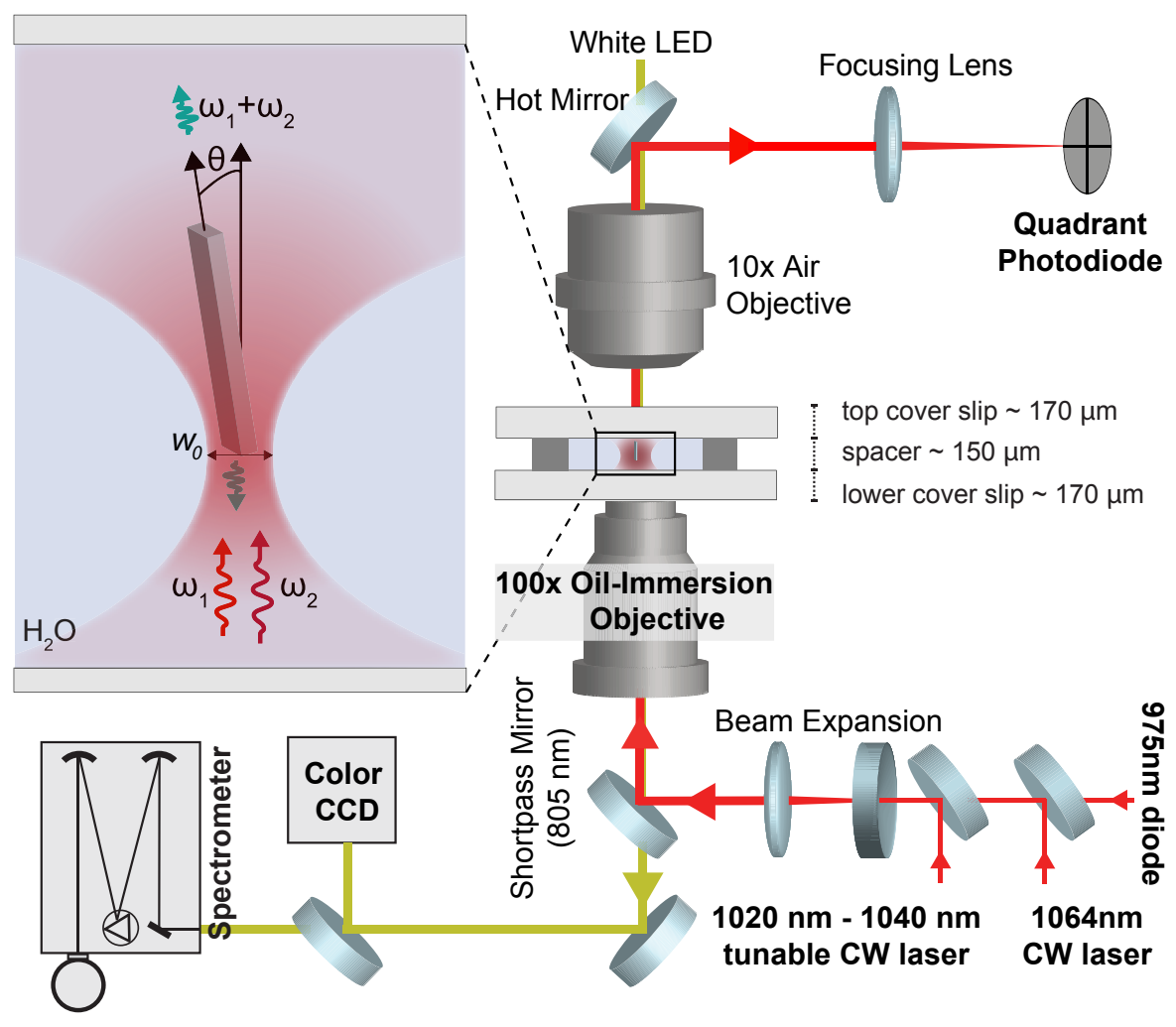

Figure 3: Schematic of experimental optical trapping setup. 

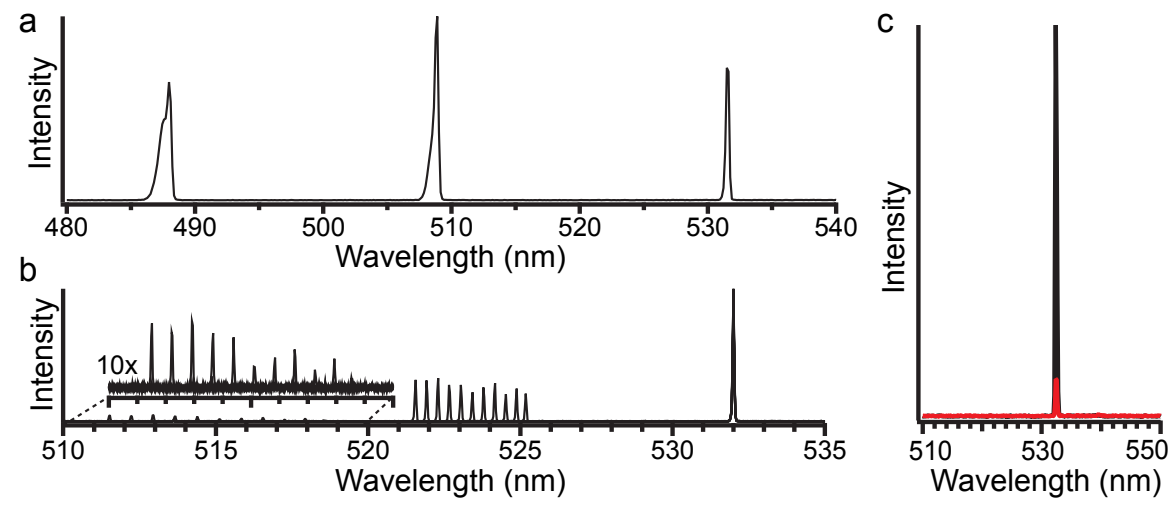

Figure 4: Sum frequency generation. a) Sum frequency generation from a KNNW using coaligned 975 and $1064 \mathrm{~nm}$ lasers. b) Tunable sum frequency generation from a KNNW using a co-aligned $1064 \mathrm{~nm}$ DPSS laser and a tunable 1020-1040 nm DPSS laser. c) SHG of $1064 \mathrm{~nm}$ fundamental laser with polarizer parallel (black) and perpendicular (red) to the fundamental polarization. 

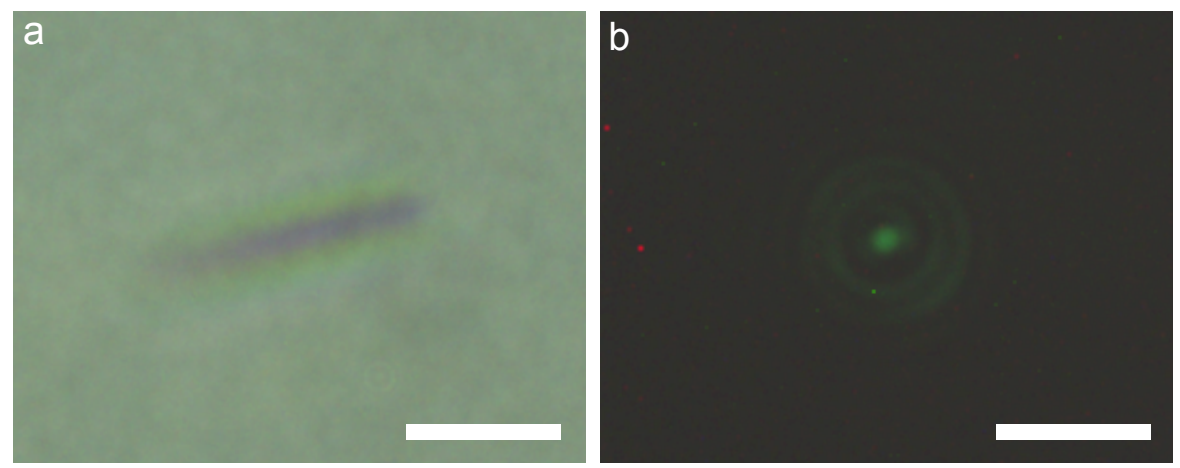

C
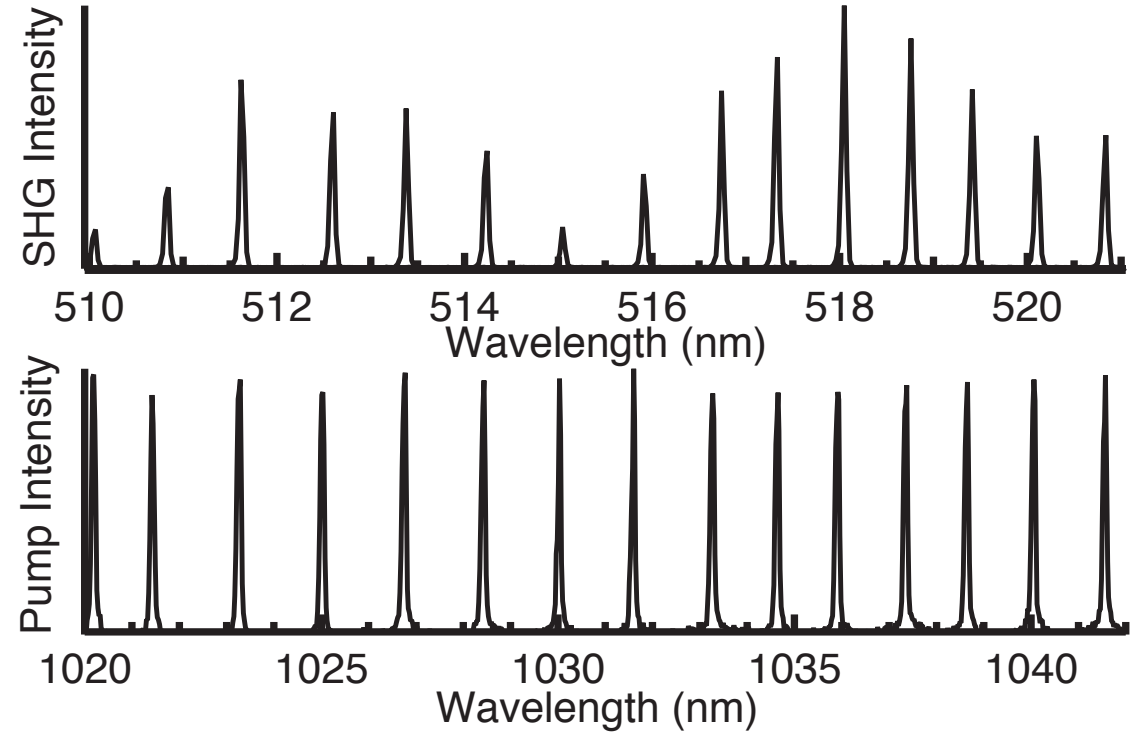

Figure 5: (a) Bright field image of a KNNW in Brownian motion. Scale bar $=2 \mu \mathrm{m}$. (b) Long-exposure, dark image demonstrating SHG from the KNNW while trapped with a 1030 nm source. Scale bar $=2 \mu \mathrm{m}$. (c) A NIR tunable laser was tuned from 1020 to $1040 \mathrm{~nm}$ while maintaining a constant trapping power (lower panel). The second harmonic generation from a KNNW was detected at each discrete pump wavelength (upper panel). The oscillations with the SHG are due to a Fabry-Pérot resonance within the NW. 


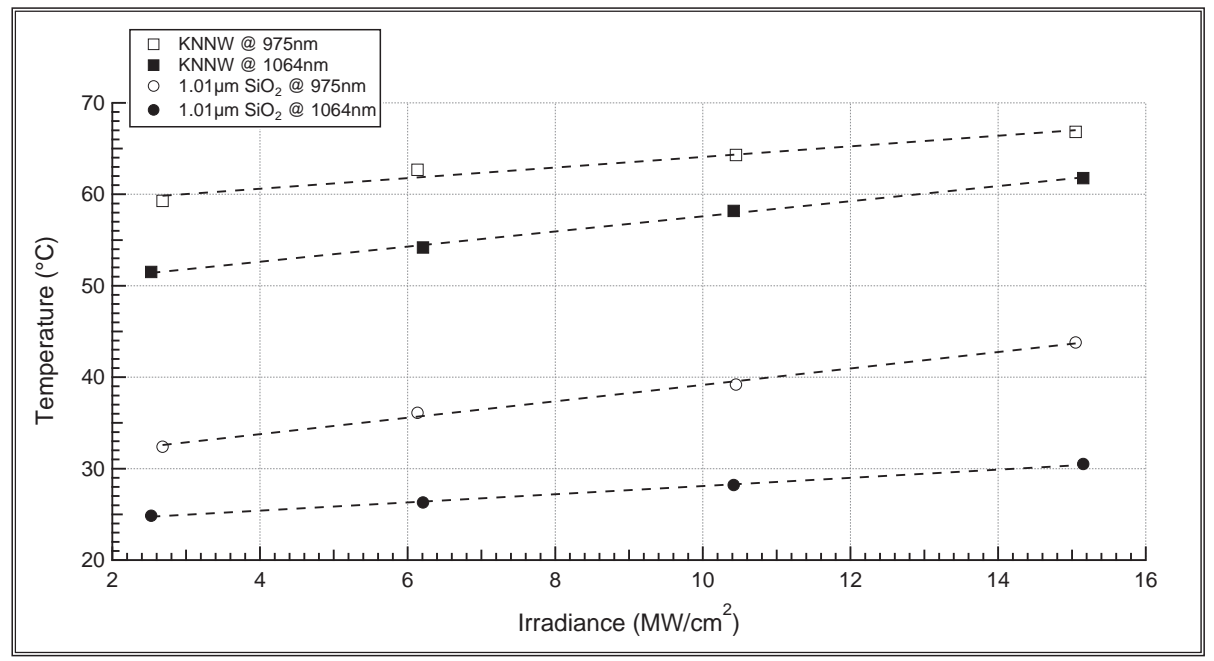

Figure 6: Temperatures of trapped KNNWs (squares) and $\mathrm{SiO}_{2}$ beads (circles) using a 975 $\mathrm{nm}$ laser diode (open) or $1064 \mathrm{~nm}$ DPSS laser (closed) measured using Brownian analysis of forward-scattered laser light. 